\title{
A HYPERSURFACE DEFECT RELATION FOR A CLASS OF MEROMORPHIC MAPS ${ }^{1}$
}

BY

\author{
ALDO BIANCOFIORE
}

\begin{abstract}
Let $D_{1}, \ldots, D_{q}$ be hypersurfaces of degree $p$ in $\mathbf{P}_{n}$ with normal crossings. We prove for a certain class of meromorphic maps $f: \mathbf{C}^{m} \rightarrow \mathbf{P}_{n}$ a defect relation $\delta_{f}\left(D_{1}\right)+\cdots+\delta_{f}\left(D_{q}\right) \leqslant(n+1) / p$ conjectured by Ph. Griffiths and B. Shiffman.
\end{abstract}

Introduction. Let $f: \mathbf{C}^{m} \rightarrow \mathbf{P}_{n}$ be a meromorphic map. Let $D_{1}, \ldots, D_{q}$ be hypersurfaces of degree $p$ in $\mathbf{P}_{n}$ such that $f\left(\mathbf{C}^{m}\right) \nsubseteq D_{j}$ for $j=1, \ldots, q$. The Nevanlinna defect $\delta_{f}\left(D_{j}\right)$ of $f$ for $D_{j}$ is defined for $j=1, \ldots, q$. When does the defect relation

$$
\sum_{j=1}^{q} \delta_{f}\left(D_{j}\right) \leqslant(n+1) / p
$$

hold? We shall provide a partial answer to this question.

If $p=1$, this is the classical defect relation (Nevanlinna [8], H. Cartan [4], Ahlfors [1], Weyl [13], Stoll [11], Vitter [12]). Here we are concerned with the case $p \geqslant 2$. The Carlson-Griffiths-King theory $[3,6]$ implies (1) if $D_{1}, \ldots, D_{q}$ have normal crossings and if $m \geqslant n=\operatorname{rank} f$. Hopefully this rank condition can be replaced by a more natural assumption which permits $m \leqslant n$.

P. Griffiths [5] conjectured that (1) holds if the image of $f$ is not contained in any hypersurface of degree $p$ and if $D_{1}, \ldots, D_{q}$ have normal crossings. We provide a counterexample (\$5). If $f\left(\mathbf{C}^{m}\right)$ is not contained in any hypersurface, the conjecture (Shiffman [9]) remains unresolved, even if $m=1$, despite many attempts.

In 1979 B. Shiffman [10] investigated a particular class $\subseteq$ of meromorphic maps of finite order. He considers $D_{1}, \ldots, D_{q}$ distinct hypersurfaces of degree $p$ such that no point of $\mathbf{P}_{n}$ is contained in $n+1$ distinct $D_{j}$. If $f \in \subseteq$ and $f\left(\mathbf{C}^{m}\right) \nsubseteq D_{j}$ for $j=1, \ldots, q$ he shows

$$
\sum_{j=1}^{q} \delta_{f}\left(D_{j}\right) \leqslant 2 n
$$

Under these assumptions, $2 n$ is the best possible bound.

We prove (1) for a more general class $\Re$ of meromorphic maps, but, naturally we impose the stricter condition of normal crossings on the divisors. The maps of our

Received by the editors May 12, 1980. Paper presented at the 784th A.M.S. Meeting, Notre Dame, March 20-21, 1981.

1980 Mathematics Subject Classification. Primary 32A22, 32H25, 32H30; Secondary 30D35.

Key words and phrases. Defect relation, Second Main Theorem.

'This research was partially supported by a grant of the Consiglio Nazionale delle Ricerche (Italy). 
class $\Re$ have infinite order or finite integral order and are "projections" of meromorphic maps with maximal linear Nevanlinna deficiencies.

In $\S 1$ and $\S 2$, we assemble the basic notions. In $\S 3$, we investigate the properties of normal crossings. The defect relation (1) is proved in $\$ 4$. In $\$ 5$ we provide a counterexample to (1), if the image of $f$ is not contained in any hypersurface of degree $p$, but is contained in a higher dimensional hypersurface. In $\S 6$ we investigate the relation of our class of maps with Shiffman class.

I wish to thank my thesis advisor, Professor W. Stoll, who introduced me to value distribution theory and placed all his knowledge at my disposal.

1. Preliminaries. Let $f: \mathbf{C}^{m} \rightarrow \mathbf{P}_{n}$ be a meromorphic map. A holomorphic vector function $\mathfrak{b}: \mathbf{C}^{m} \rightarrow \mathbf{C}^{n+1}$ is said to be a representation for $f$ if $\mathfrak{b}^{-1}(0) \neq \mathbf{C}^{m}$ and $\mathbf{P} \circ \mathfrak{v}=f$ on $\mathbf{C}^{m}-\mathfrak{v}^{-1}(0)$. The representation is said to be reduced if $\operatorname{dim} \mathfrak{v}^{-1}(0) \leqslant m$ -2 .

A meromorphic map $f: \mathbf{C}^{m} \rightarrow \mathbf{P}_{n}$ is said to be nondegenerate of degree $p$ if for any hypersurface $D$ of degree $p$ in $\mathbf{P}_{n}$ we have $f\left(\mathbf{C}^{m}\right) \nsubseteq \operatorname{supp} D$, otherwise $f$ is said to be degenerate of degree $p$. If a meromorphic map is nondegenerate (or degenerate) of degree 1 we say that $f$ is nondegenerate (or degenerate).

2. Nevanlinna theory. If $z=\left(z_{1}, \ldots, z_{m}\right) \in \mathbf{C}^{m}$, define

$$
|z|=\left(\left|z_{1}\right|^{2}+\cdots+\left|z_{m}\right|^{2}\right)^{1 / 2} \text { and } \tau(z)=|z|^{2} .
$$

If $r>0$, set $\mathbf{C}^{m}[r]=\left\{z \in \mathbf{C}^{m}|| z \mid \leqslant r\right\}$ and $\mathbf{C}^{m}\langle r\rangle=\partial \mathbf{C}^{m}[r]$. We define

$$
\begin{aligned}
& v=d d^{c} \tau \quad \text { on } \mathbf{C}^{m}, \\
& \boldsymbol{\sigma}=\sigma_{m}=d^{c} \log \tau \wedge\left(d d^{c} \log \tau\right)^{m-1} \text { on } \mathbf{C}^{m}-\{0\},
\end{aligned}
$$

where $d^{c}=(i / 4 \pi)(\bar{\partial}-\partial)$.

Let $\nu$ be a divisor on $\mathbf{C}^{m}$. Abbreviate $S[r]=\mathbf{C}^{m}[r] \cap \operatorname{supp} \nu$, if $0<r \in \mathbf{R}$, the counting function of $\nu$ is defined by

$$
n_{\nu}(r)= \begin{cases}r^{2-2 m} \int_{S[r]} \nu v^{m-1} & \text { if } m>1 \\ \sum_{z \in S[r]} \nu(z) & \text { if } m=1 .\end{cases}
$$

For all $0<r_{0}<r$ the valence function of $\nu$ is defined by

$$
N_{\nu}\left(r, r_{0}\right)=\int_{r_{0}}^{r} n_{\nu}(t) t^{-1} d t .
$$

Let $f: \mathbf{C}^{m} \rightarrow \mathbf{P}_{n}$ be a meromorphic map. Let $\omega$ be the Fubini-Kaehler form on $\mathbf{P}_{n}$. For $r>r_{0}>0$, define the characteristic function of $f$

$$
T_{f}\left(r, r_{0}\right)=\int_{r_{0}}^{r} t^{1-2 m} d t \int_{\mathbf{C}^{m}[r]} f^{*}(\omega) \wedge v^{m-1}
$$

and for any hypersurface of degree $p$ in $\mathbf{P}_{n}$ we define the valence function of $f$ for $D$

$$
N_{f}\left(r, r_{0}, D\right)=N_{\nu_{f}^{p}}\left(r, r_{0}\right) \geqslant 0,
$$

where $\nu_{f}^{D}=f^{*}(D)$ is the pull-back divisor. 
We shall need the following well-known results. Let $D$ be a hypersurface of degree $p$ on $\mathbf{P}_{n}$ such that $f\left(\mathbf{C}^{m}\right) \nsubseteq \operatorname{supp} D$. Let $\alpha$ be a homogeneous polynomial of degree $p$ on $\mathbf{C}^{n+1}$ such that $\mathbf{P}\left(\alpha^{-1}(0)\right)=\operatorname{supp} D$. Then for $r>r_{0}>0$ we have

(2.1) First Main Theorem.

$$
p T_{f}\left(r, r_{0}\right)+O(1) \geqslant N_{f}\left(r, r_{0}, D\right) .
$$

(2.2) JENSEN'S FORMULA.

$$
\begin{gathered}
N_{f}\left(r, r_{0}, D\right)=\int_{\mathbf{C}^{m}\langle r\rangle} \log |\alpha \circ \mathfrak{v}| \sigma-\int_{\mathbf{C}^{m}\left\langle r_{0}\right\rangle} \log |\alpha \circ \mathfrak{v}| \sigma . \\
T_{f}\left(r, r_{0}\right)=\int_{\mathbf{C}^{m}\langle r\rangle} \log |\mathfrak{v}| \sigma-\int_{\mathbf{C}^{m}\left\langle r_{0}\right\rangle} \log |\mathfrak{v}| \sigma,
\end{gathered}
$$

where $\mathfrak{b}$ denotes a reduced representation of $f$.

Let $D$ be a hypersurface of degree $p$ and assume that $f\left(\mathbf{C}^{m}\right) \nsubseteq \operatorname{supp} D$. The defect of $f$ for the hypersurface $D$ is defined by

$$
\delta_{f}(D)=\liminf _{r \rightarrow \infty}\left(1-\frac{N_{f}\left(r, r_{0}, D\right)}{p T_{f}\left(r, r_{0}\right)}\right) .
$$

By (2.1) we have

$$
0 \leqslant \delta_{f} \leqslant 1 .
$$

We shall use from now on the following notation. Let $g$ and $h$ be real valued functions on $\mathbf{R}\left(r_{0}, \infty\right)$. We write $g(r) \leqslant h(r)$ if there exists a subset $E$ of $\mathbf{R}\left(r_{0}, \infty\right)$ with finite Lebesgue measure such that $g(r) \leqslant h(r)$ for all $r \in \mathbf{R}\left(r_{0}, \infty\right)-E$.

Now we can state

Second Main Theorem (Stoll [11]). Let $f: \mathbf{C}^{m} \rightarrow \mathbf{P}_{n}$ be a nondegenerate, meromorphic map. Let $H_{1}, \ldots, H_{q}$ be hyperplanes in $\mathbf{P}_{n}$ in general position with $q \geqslant n+1$. Then

$$
(q-n-1) T_{f}\left(r, r_{0}\right) \leqslant \sum_{j=1}^{q} N_{f}\left(r, r_{0}, H_{j}\right)+O\left(\log r T_{f}\left(r, r_{0}\right)\right) .
$$

3. Normal crossings. Let $D$ be a hypersurface of degree $p$ in $\mathbf{P}_{n}$. Let $\alpha$ be a homogeneous polynomial of degree $p$ on $\mathbf{C}^{n+1}$ such that $\mathbf{P}\left(\alpha^{-1}(0)\right)=\operatorname{supp} D$. Then we set $D=D[\alpha]$.

Let $D_{j}=D\left[\alpha_{j}\right]$ be hypersurfaces of degree $p$ on $\mathbf{P}_{n}$, for $j=1, \ldots, q$. For $y=\mathbf{P}(\mathfrak{h})$ $\in \mathbf{P}_{n}$, let

$$
d=d(y)=\#\left\{j \in \mathbf{N}[1, q] \mid \boldsymbol{\alpha}_{j}(\mathfrak{h})=0\right\} .
$$

We have $0 \leqslant d \leqslant q$. Call $d=d(y)$ the crossings number of $D_{1}, \ldots, D_{q}$ at $y$. If $d(y) \geqslant 1$ then there exists one and only one $\kappa=\kappa(y): \mathbf{Z}[1, d] \rightarrow \mathbf{Z}[1, q]$ such that $\alpha_{\kappa(j)}(\mathfrak{h})=0$ for every $j=1, \ldots, d$. Call $\kappa$ the crossings selector of $D_{1}, \ldots, D_{q}$ at $y$ and

$$
J(y)=J\left(D_{1}, \ldots, D_{q}, y\right)=d \alpha_{\kappa(1)}(\mathfrak{h}) \wedge \cdots \wedge d \alpha_{\kappa(d)}(\mathfrak{h})
$$


the crossings Jacobian of $D_{1}, \ldots, D_{q}$ at $y$ (here $d \alpha_{j}(\mathfrak{h})$ is considered as an element of $\left.\left(\mathbf{C}^{n+1}\right)^{*}\right)$.

We say that $D_{1}, \ldots, D_{q}$ have normal crossings at $y$ if and only if $d(y) \geqslant 1$ and $J(y) \neq 0$. Also we say that $D_{1}, \ldots, D_{q}$ have normal crossings if and only if $D_{1}, \ldots, D_{q}$ have normal crossings at every $y \in \cup_{j=1}^{q} \operatorname{supp} D_{j}$.

Remark 3.1. (a) If $D_{1}, \ldots, D_{q}$ have normal crossings at $y$ then $d(y) \leqslant n$.

(b) If $D_{1}, \ldots, D_{q}$ have normal crossings then $D_{j}$ is smooth for $j=1, \ldots, q$.

Lemma 3.2. Let $\varphi: \mathbf{C}^{N+1} \rightarrow \mathbf{C}^{n+1}$ be a surjective linear map. Take $\mathfrak{h} \in \mathbf{C}^{N+1}-$ ker $\varphi$. Let $D_{j}=D\left[\alpha_{j}\right]$ be hypersurfaces of degree $p$ in $\mathbf{P}_{n}$, for $j=1, \ldots, q$. Set $\tilde{D}_{j}=D\left[\alpha_{j} \circ \varphi\right]$. Then $D_{1}, \ldots, D_{q}$ have normal crossings at $\mathbf{P}(\varphi(\mathfrak{h}))$ with crossings number $d$ and crossings selector $\kappa$ if and only if $\tilde{D}_{1}, \ldots, \tilde{D}_{q}$ have normal crossings at $\mathbf{P}(\mathfrak{h})$ with crossings number $d$ and crossings selector $\kappa$.

Proof. By definition the crossings number and the crossings selector are the same. Set $\beta_{j}=\alpha_{j} \circ \varphi$. We have

$$
d \beta_{j}(\mathfrak{h})=d \alpha_{j}(\varphi(\mathfrak{h})) \circ d \varphi(\mathfrak{h})=d \alpha_{j}(\varphi(\mathfrak{h})) \circ \varphi .
$$

So if $y=\mathbf{P}(\mathfrak{h})$ and $x=\mathbf{P}(\varphi(\mathfrak{h}))$

$$
\begin{aligned}
J\left(\tilde{D}_{1}, \ldots, \tilde{D}_{q}, y\right) & =d \beta_{\kappa(1)}(\mathfrak{h}) \wedge \cdots \wedge d \beta_{\kappa(d)}(\mathfrak{h}) \\
& =d \alpha_{\kappa(1)}(\varphi(\mathfrak{h})) \circ \varphi \wedge \cdots \wedge d \alpha_{\kappa(d)}(\varphi(\mathfrak{h})) \circ \varphi \\
& =\varphi^{*}\left(d \alpha_{\kappa(1)}(\varphi(\mathfrak{h})) \wedge \cdots \wedge d \alpha_{\kappa(d)}(\varphi(\mathfrak{h}))\right) \\
& =\varphi^{*}\left(J\left(D_{1}, \ldots, D_{q}, x\right)\right) .
\end{aligned}
$$

Since $\varphi$ is surjective, $\varphi^{*}$ is injective. Hence $J\left(\tilde{D}_{1}, \ldots, \tilde{D}_{q}, y\right) \neq 0$ if and only if $J\left(D_{1}, \ldots, D_{q}, x\right) \neq 0$. Q.E.D.

Let $\zeta_{j}: \mathbf{C}^{N+1} \rightarrow \mathbf{C}$ be the $j$ th coordinate function, i.e. $\zeta_{j}\left(z_{0}, \ldots, z_{N}\right)=z_{j}$. Set

$$
e_{j}=e_{j}^{(N)}=(\underbrace{0, \ldots, 0}_{j}, 1,0, \ldots, 0) \in \mathbf{C}^{N+1} .
$$

If $J: \mathbf{Z}[0, N] \rightarrow \mathbf{Z}[0, p]$ let $J(k)=j_{k}$ and $J=\left(j_{0}, \ldots, j_{N}\right)$. Let $K=K(p)$ be the set of all $J: \mathbf{Z}[0, N] \rightarrow \mathbf{Z}[0, p]$ such that $j_{0}+\cdots+j_{N}=p$. If $J \in K$ then $\zeta^{J}=\zeta_{0}^{j_{0}}$ $\cdots \zeta_{N}^{j_{N}}: \mathbf{C}^{N+1} \rightarrow \mathbf{C}$ is a homogeneous polynomial of degree $p$. Then if we denote by $\mathbf{C}_{(p)}^{N+1}$ all the homogeneous polynomials of degree $p$ on $\mathbf{C}^{N+1}$ we have that $\left\{\zeta^{J} \mid J \in\right.$ $K\}$ is a base for $\mathbf{C}_{(p)}^{N+1}$ over $\mathbf{C}$. Therefore if $\alpha \in \mathbf{C}_{(p)}^{N+1}$ then $\alpha=\Sigma_{J \in K} \alpha_{J} \zeta^{J}$ where the coefficients $\alpha_{J} \in \mathbf{C}$ are unique. We set $B=B(p)=\left\{J \in K(p) \mid j_{k} \geqslant p-1\right.$ for some $k \in \mathbf{Z}[0, N]\}$. Consider the map $\delta=\delta_{p}:(\mathbf{Z}[0, N])^{2} \rightarrow B$, for $p \geqslant 2$ defined as follows:

$$
\delta(h, k)(j)= \begin{cases}0 & \text { if } h \neq j \neq k \\ 1 & \text { if } h=j \neq k \\ p-1 & \text { if } h \neq j=k \\ p & \text { if } h=j=k\end{cases}
$$


Define $\varepsilon=\varepsilon_{p}:(\mathbf{Z}[0, N])^{2} \rightarrow\{1,2\}$ by $\varepsilon(h, k)=\# \delta^{-1}(\delta(h, k))$. Then we have $\varepsilon(h, k)$ $\equiv 1$ if $p>2$ and for $p=2$

$$
\varepsilon(h, k)= \begin{cases}1 & \text { if } h=k \\ 2 & \text { if } h \neq k\end{cases}
$$

If $\alpha=\Sigma_{J \in K} \alpha_{J} \zeta^{J} \in \mathbf{C}_{(p)}^{N+1}$, we set

$$
\begin{array}{r}
\rho(\alpha)=\sum_{J \in B} \alpha_{J} \zeta^{J}=\sum_{h, k=0}^{N} \alpha_{h k} \zeta_{h} \zeta_{k}^{p^{-1}} \in \mathbf{C}_{(p)}^{N+1}, \\
\rho_{k}(\alpha)=\sum_{h=0}^{N} \alpha_{h k} \zeta_{h} \in \mathbf{C}_{(1)}^{N+1} \text { for } k=0, \ldots, N,
\end{array}
$$

where $\alpha_{h k}=\varepsilon(h, k)^{-1} \alpha_{\delta(h, k)}$. We have

$$
\rho(\alpha)=\sum_{k=0}^{N} \rho_{k}(\alpha) \zeta_{k}^{p-1} .
$$

LeMma 3.3. Let $\alpha \in \mathbf{C}_{(p)}^{N+1}$. Then, for $k \in \mathbf{Z}[0, N]$

(i) $\rho(\alpha)\left(e_{k}\right)=\alpha\left(e_{k}\right)=\rho_{k}(\alpha)\left(e_{k}\right)$.

(ii) $(d \rho(\alpha))\left(e_{k}\right)=d \alpha\left(e_{k}\right)$.

(iii)

$$
d \alpha\left(e_{k}\right)= \begin{cases}2 \rho_{k}(\alpha) & \text { if } p=2 \\ \rho_{k}(\alpha) & \text { if } p>2 \text { and } \alpha\left(e_{k}\right)=0 .\end{cases}
$$

Proof. (i) Set $\chi=\alpha-\rho(\alpha)$. We have $\chi\left(e_{k}\right)=0, d \chi\left(e_{k}\right)=0$ and $\rho(\chi)=\rho_{k}(\chi)$ $=0$ for $k=0, \ldots, N$. If $\rho(\alpha)=\sum_{h, k=0}^{N} \alpha_{h k} \zeta_{h} \zeta_{k}^{-1}$ then $\alpha\left(e_{k}\right)=\rho(\alpha)\left(e_{k}\right)=\alpha_{k k}=$ $\rho_{k}(\alpha)\left(e_{k}\right)$.

(ii) It is a direct consequence of $d \chi\left(e_{k}\right)=0$.

(iii) We have

$$
(d \rho(\alpha))\left(e_{k}\right)= \begin{cases}p \alpha_{k k} \zeta_{k}+\Sigma_{h \neq k} \alpha_{h k} \zeta_{k} & \text { if } p>2, \\ 2 \rho_{k}(\alpha) & \text { if } p=2 .\end{cases}
$$

Therefore by (ii), if $p=2$ we have $d \alpha\left(e_{k}\right)=2 \rho_{k}(\alpha)$, if $p>2$ and $\alpha\left(e_{k}\right)=\alpha_{k k}=0$ then $d \alpha\left(e_{k}\right)=\rho^{k}(\alpha)$. Q.E.D.

Let $D_{j}=D\left[\alpha_{j}\right]$ be hypersurfaces of degree $p$ for $j=1, \ldots, q$. Set $R_{j}=D\left[\rho\left(\alpha_{j}\right)\right]$ and $R_{k j}=D\left[\rho_{k}\left(\alpha_{j}\right)\right]$. Let $\varphi: \mathbf{C}^{N+1} \rightarrow \mathbf{C}^{n+1}$ be a surjective linear map. Set $\tilde{D}_{j}=$ $D\left[\alpha_{j} \circ \varphi\right], \tilde{R}_{j}=D\left[\rho\left(\alpha_{j} \circ \varphi\right)\right]$ and $\tilde{R}_{k j}=D\left[\rho_{k}\left(\alpha_{j} \circ \varphi\right)\right]$ for $j=1, \ldots, q$. Then as a consequence of Lemma 3.2 and Lemma 3.3 we get

Corollary 3.4. (a) $\left\{D_{j}\right\},\left\{R_{j}\right\}$ and $\left\{R_{k j}\right\}$ have the same crossings number and crossings selector at $\mathbf{P}\left(e_{k}\right)$. Moreover if one of them has normal crossings at $\mathbf{P}\left(e_{k}\right)$ then all of them have normal crossings at $\mathbf{P}\left(e_{k}\right)$.

(b) If $\left\{D_{j}\right\}$ has normal crossings at $\mathbf{P}\left(\varphi\left(e_{k}\right)\right)$ with crossings number $d$ and crossings selector $\kappa$, then $\left\{\tilde{D}_{j}\right\},\left\{\tilde{R}_{j}\right\}$ and $\left\{\tilde{R}_{k j}\right\}$ have normal crossings at $\mathbf{P}\left(e_{k}\right)$ with crossings number $d$ and crossings selector $\kappa$. 
Let $\eta_{0}: \mathbf{C} \rightarrow\{0,1\}$ be defined by $\eta_{0}(a)=1$ if $a \neq 0$ and $\eta_{0}(0)=0$. If $\alpha=$ $\Sigma_{J \in K} \alpha_{J} \zeta^{J} \in \mathbf{C}_{(p)}^{N+1}$ we define

$$
\eta_{p}(\alpha)=\sum_{J \in K} \eta_{0}\left(\alpha_{J}\right)\left|\zeta^{J}\right|
$$

Proposition 3.5. Let $\varphi: \mathbf{C}^{N+1} \rightarrow \mathbf{C}^{n+1}$ be a surjective linear map. Let $D_{j}=D\left[\alpha_{j}\right]$ be hypersurfaces of degree $p$ in $\mathbf{P}_{n}$, for $j=1, \ldots, q$. Assume that $D_{1}, \ldots, D_{q}$ have normal crossings. Then there exist a constant $c>0$ and an injective map $\tau: \mathbf{Z}[0, n] \rightarrow \mathbf{Z}[0, N]$ such that

$$
\prod_{j=1}^{q} \eta_{p}\left(\alpha_{j} \circ \varphi\right) \geqslant c|\zeta|^{q p-n-1} \prod_{h=0}^{n}\left|\zeta_{\tau(h)}\right|,
$$

where $|\zeta|=\left(\sum_{h=0}^{N}\left|\zeta_{h}\right|^{2}\right)^{1 / 2}$.

Proof. We set $\beta_{j}=\alpha_{j} \circ \varphi$ for $j=1, \ldots, q, \gamma=\eta_{p} \circ \rho$ and $\gamma_{k}=\eta_{1} \circ \rho_{k}$. Then by (3.1) we have

$$
\gamma\left(\beta_{j}\right)=\sum_{k=0}^{N} \gamma_{k}\left(\beta_{j}\right)\left|\zeta_{k}\right|^{p-1} \text { for } j=1, \ldots, q
$$

and

$$
\eta_{p}\left(\beta_{j}\right) \geqslant \gamma\left(\beta_{j}\right)=\eta_{p}\left(\rho\left(\beta_{j}\right)\right)
$$

So

$$
\begin{aligned}
\prod_{j=1}^{q} \eta_{p}\left(\beta_{j}\right) & \geqslant \prod_{j=1}^{q} \gamma\left(\beta_{j}\right)=\prod_{j=1}^{q} \cdot \sum_{h=0}^{N} \gamma_{h}\left(\beta_{j}\right)\left|\zeta_{h}\right|^{p-1} \\
& \geqslant \sum_{h=0}^{N}\left(\prod_{j=1}^{q} \gamma_{h}\left(\beta_{j}\right)\right)\left|\zeta_{h}\right|^{q p-q} .
\end{aligned}
$$

Since $\varphi$ is surjective, there exists $\tau: \mathbf{Z}[0, n] \rightarrow \mathbf{Z}[0, N]$ such that $\left\{\varphi\left(e_{\tau(0)}\right)\right.$, $\left.\ldots, \varphi\left(e_{\tau(n)}\right)\right\}$ is a base for $\mathbf{C}^{n+1}$. Set $T=\tau(\mathbf{Z}[0, n]) \subseteq \mathbf{Z}[0, N]$ and $T_{h}=T-\{h\}$.

Claim 1. Let $d=d_{h}$ be the crossings number of $D_{1}, \ldots, D_{q}$ at $\mathbf{P}\left(\varphi\left(e_{h}\right)\right)$ then there exists $\varepsilon=\varepsilon_{h}: \mathbf{Z}\left[1, d_{h}\right] \rightarrow T_{h}$ such that

$$
\prod_{j=1}^{q} \gamma\left(\beta_{j}\right) \geqslant\left|\zeta_{\varepsilon(1)}\right| \cdots\left|\zeta_{\varepsilon(d)}\right|\left|\zeta_{h}\right|^{q-d}
$$

Proof of Claim 1. By definition we have $\gamma_{h}\left(\beta_{j}\right)=\eta_{1}\left(\rho_{h}\left(\beta_{j}\right)\right)$. Let $\kappa=\kappa_{h}$ be the crossings selector of $D_{1}, \ldots, D_{q}$ at $\mathbf{P}\left(\varphi\left(e_{h}\right)\right)$. Then by Corollary $3.4(\mathrm{~b}), \tilde{R}_{h j}=$ $D\left[\rho_{h}\left(\beta_{j}\right)\right]$ for $j=1, \ldots, q$ have normal crossings at $\mathbf{P}\left(e_{h}\right)$ with crossings number $d$ and crossings selector $\kappa$. Set $\chi_{j}=\rho_{h}\left(\beta_{\kappa(j)}\right)$. Then we have

$$
\prod_{i=1}^{q} \gamma_{h}\left(\beta_{i}\right)=\left(\prod_{j=1}^{d} \eta_{1}\left(\chi_{j}\right)\right)\left|\zeta_{h}\right|^{q-d}
$$


Now $\chi_{1} \wedge \cdots \wedge \chi_{d} \neq 0, \chi_{j}\left(e_{h}\right)=0$ for $j=1, \ldots, d,\left\{\varphi\left(e_{\tau(0)}\right), \ldots, \varphi\left(e_{\tau(n)}\right)\right\}$ a base for $\mathbf{C}^{n+1}$ and an easy argument of linear algebra show that there exists $\varepsilon=\varepsilon_{h}$ : $\mathbf{Z}[1, d] \rightarrow T_{h}$ injective such that

$$
\prod_{j=1}^{d} \eta_{1}\left(\chi_{j}\right) \geqslant\left|\zeta_{\varepsilon(1)}\right| \cdots\left|\zeta_{\varepsilon(d)}\right|
$$

Therefore we get (3.4).

From (3.3) and (3.4) we obtain

$$
\prod_{j=1}^{q} \eta_{p}\left(\beta_{j}\right) \geqslant \sum_{h=0}^{N}\left|\zeta_{\varepsilon_{h}(1)}\right| \cdots\left|\zeta_{\varepsilon_{h}\left(d_{h}\right)}\right|\left|\zeta_{h}\right|^{q p-d_{h}}
$$

Abbreviate

$$
f_{h}=\left|\zeta_{\varepsilon_{h}(1)}\right| \cdots\left|\zeta_{\varepsilon_{h}\left(d_{h}\right)}\right|\left|\zeta_{h}\right|^{q p-d_{h}}, \quad g_{h}=\left(\prod_{j=0}^{n}\left|\zeta_{\tau(j)}\right|\right)\left|\zeta_{h}\right|^{q p-n-1}
$$

and

$$
f=\sum_{h=0}^{N} f_{h}, \quad g=\sum_{h=0}^{N} g_{h}
$$

Claim 2.

$$
f \leqslant g /(N+1) .
$$

Proof of Claim 2. W.l.o.g. we may assume $\tau(j)=j$ and therefore $\varepsilon_{h}\left(\mathbf{Z}\left[1, d_{h}\right]\right) \subseteq$ $\mathbf{Z}[0, n]$. We set $x_{j}=\left|\zeta_{j}(z)\right| \in \mathbf{R}_{+}$for $z \in \mathbf{C}^{N+1}$ and $j=0, \ldots, N$. Let $j_{0}, \ldots, j_{N}$ be a permutation of $\{0, \ldots, N\}$ such that $x_{j_{0}} \geqslant \cdots \geqslant x_{j_{N}}$. Abbreviate $k=j_{0}$. Then

$$
\begin{aligned}
(N+1)^{-1} g(z) & \leqslant \operatorname{Max}_{0 \leqslant j \leqslant N} g_{j}(z)=x_{0} \cdots x_{n} x_{k}^{q p-n-1} \\
& =x_{0} \cdots x_{n} x_{k}^{d_{k}-n-1} x_{k}^{q p-d_{k}} \\
& \leqslant x_{\varepsilon_{k}(1)} \cdots x_{\varepsilon_{k}\left(d_{k}\right)} x_{k}^{q p-d_{k}}=f_{k}(z) \leqslant f(z) .
\end{aligned}
$$

Using (3.5), (3.6) and the inequalities

$$
(N+1)^{(n+2-q p)}\left(\sum_{j=0}^{N}\left|\zeta_{j}\right|^{q p-n-1}\right) \leqslant \sum_{j=0}^{N}\left|\zeta_{j}\right|^{q p-n-1} \leqslant \sum_{j=0}^{N}\left|\zeta_{j}\right|^{q p-n-1}
$$

we get (3.2) where $c=(N+1)^{n+1-q p}$. Q.E.D.

4. Defect relation. Let $\zeta_{j}: \mathrm{C}^{N+1} \rightarrow \mathrm{C}$ be the $j$ th coordinate function. Set $H_{j}=$ $\mathbf{P}\left(\zeta_{j}^{-1}(0)\right) \subseteq \mathbf{P}_{N}$. If $\varphi: \mathbf{C}^{N+1} \rightarrow \mathbf{C}^{n+1}$ is a linear map we denote by $\mathbf{P}(\varphi)$ the projective map induced by $\varphi$. Abbreviate

$$
e_{j}=(\underbrace{0, \ldots, 0}_{j}, 1,0, \ldots, 0) \text { for } j=0, \ldots, N .
$$


Definition 4.1. Let $f: \mathbf{C}^{m} \rightarrow \mathbf{P}_{n}$ be a meromorphic map. We say that $f \in \mathfrak{D}$, if there exist a meromorphic map $g: \mathbf{C}^{m} \rightarrow \mathbf{P}_{N}$ and a linear map $\varphi: \mathbf{C}^{N+1} \rightarrow \mathbf{C}^{n+1}$ such that

1. $f=\mathbf{P}(\varphi) \circ g$.

2. $\delta_{g}\left(H_{j}\right)=1$ or $N_{g}\left(r, r_{0}, H_{j}\right)=o\left(T_{g}\left(r, r_{0}\right)\right), j=0, \ldots, N$.

3. If $\mathfrak{v}$ and $\mathfrak{g}$ are reduced representations of $f$ and $g$ respectively, let $u$ : $\mathbf{C}^{m} \rightarrow \mathbf{C}$ be a holomorphic function such that $u \mathrm{v}=\varphi \circ \mathrm{g}$, then we require that

$$
N_{u}\left(r, r_{0}, 0\right)=o\left(T_{g}\left(r, r_{0}\right)\right) \text {. }
$$

We say that $(g, \varphi)$ satisfying conditions 1,2 and 3 is a decomposition of $f$.

REMARK 4.2. A meromorphic map $g$ satisfying condition 2 in Definition 4.1 is transcendental (see Mori [7]).

Proposition 4.3. Let $(g, \varphi)$ be a decomposition of $f \in \mathfrak{D}$. Then

$$
T_{f}\left(r, r_{0}\right) \leqslant T_{g}\left(r, r_{0}\right)+O(1) .
$$

If, in addition, $g$ is nondegenerate and $\varphi\left(e_{j}\right) \neq 0$ for $j=0, \ldots, N$, then

$$
T_{g}\left(r, r_{0}\right) \leqslant T_{f}\left(r, r_{0}\right)+o\left(T_{f}\left(r, r_{0}\right)\right) .
$$

Proof. Let $\mathfrak{b}$ and $g$ be reduced representations of $f$ and $g$ respectively and $u$ a holomorphic function such that $u \mathfrak{v}=\varphi \circ \mathfrak{g}$. Put $\mathfrak{m}=u \mathfrak{v}=\varphi \circ \mathrm{g}$. Take $0 \neq \beta$ : $\mathbf{C}^{n+1} \rightarrow \mathbf{C}$ linear function with $|\beta|=1$. Define $\chi=\beta \circ \varphi: \mathbf{C}^{N+1} \rightarrow \mathbf{C}$ and $F=$ $u(\beta \circ \mathfrak{b})=\beta \circ \mathfrak{w}=\chi \circ \mathfrak{g}$. Schwarz's inequality implies

$$
|F| \leqslant|\beta||\mathfrak{w}|=|\mathfrak{m}|=|\varphi \circ \mathrm{g}| \leqslant|\varphi||\mathfrak{g}|
$$

and

$$
\begin{aligned}
\int_{\mathbf{C}^{m}\langle r\rangle} \log |F| \sigma & \leqslant \int_{\mathbf{C}^{m}\langle r\rangle} \log |\mathfrak{w}| \boldsymbol{\sigma}=\int_{\mathbf{C}^{m}\langle r\rangle} \log |u| \boldsymbol{\sigma}+\int_{\mathbf{C}^{m}\langle r\rangle} \log |\mathfrak{b}| \boldsymbol{\sigma} \\
& \leqslant \int_{\mathbf{C}^{m}\langle r\rangle} \log |\mathrm{g}| \boldsymbol{\sigma}+O(1) .
\end{aligned}
$$

Therefore we get

$$
\begin{aligned}
\int_{\mathbf{C}^{m}\langle r\rangle} \log |F| \boldsymbol{\sigma} & \leqslant N_{u}\left(r, r_{0}, 0\right)+T_{f}\left(r, r_{0}\right)+O(1) \\
& \leqslant T_{g}\left(r, r_{0}\right)+O(1)
\end{aligned}
$$

which implies (4.1).

In order to prove (4.2), consider $\beta$ in such a way that $\chi\left(e_{j}\right) \neq 0$ for $j=0, \ldots, N$.

Set $H_{N+1}=\mathbf{P}(\operatorname{Ker} \chi) \subseteq \mathbf{P}_{N}$. Then $H_{0}, \ldots, H_{N+1}$ are in general position and by (2.4) we have

$$
T_{g}\left(r, r_{0}\right) \leqslant \sum_{j=0}^{N+1} N_{g}\left(r, r_{0}, H_{j}\right)+O\left(\log r T_{g}\left(r, r_{0}\right)\right)
$$

But

$$
N_{g}\left(r, r_{0}, H_{j}\right)=o\left(T_{g}\left(r, r_{0}\right)\right) \text { for } j=0, \ldots, N
$$


and

$$
\begin{aligned}
N_{g}\left(r, r_{0}, H_{N+1}\right) & =\int_{\mathbf{C}^{m}\langle r\rangle} \log |\chi \circ g| \sigma+O(1)=\int_{\mathbf{C}^{m}\langle r\rangle} \log |F| \sigma+O(1) \\
& \leqslant T_{f}\left(r, r_{0}\right)+N_{u}\left(r, r_{0}, 0\right)+O(1)=T_{f}\left(r, r_{0}\right)+o\left(T_{g}\left(r, r_{0}\right)\right) .
\end{aligned}
$$

Therefore by Remark 4.2 we get

$$
T_{g}\left(r, r_{0}\right) \leqslant T_{f}\left(r, r_{0}\right)+o\left(T_{g}\left(r, r_{0}\right)\right) .
$$

Now, for every $0<\varepsilon<1$, we have

$$
(1-\varepsilon) T_{g}\left(r, r_{0}\right) \leqslant T_{f}\left(r, r_{0}\right)
$$

or

$$
o\left(T_{g}\left(r, r_{0}\right)\right) \leqslant o\left(T_{f}\left(r, r_{0}\right)\right) .
$$

Hence we get (4.2). Q.E.D.

Definition 4.4. Let $(g, \varphi)$ be a decomposition of $f \in \mathfrak{D}$. We say that $(g, \varphi)$ is a reduced decomposition if $g$ is nondegenerate and $\varphi\left(e_{j}\right) \neq 0$ for $j=0, \ldots, N$. Denote by $\Re$ the class of all meromorphic maps $f \in \mathfrak{D}$ such that $f$ admits a reduced decomposition.

We shall use the following general assumptions.

(A1) Let $f: \mathbf{C}^{m} \rightarrow \mathbf{P}_{n}$ be a meromorphic map. Assume $f \in \Re$ with $(g, \varphi)$ as a reduced decomposition.

(A2) Let $\mathfrak{b}$ and $g$ be reduced representations of $f$ and $g$ respectively.

(A3) Let $u: \mathbf{C}^{m} \rightarrow \mathbf{C}$ be a holomorphic function such that $u \mathfrak{b}=\varphi \circ g$ and $N_{u}\left(r, r_{0}, 0\right)=o\left(T_{g}\left(r, r_{0}\right)\right)$.

LEMMA 4.5. Assume that (A1)-(A3) holds. Let $D=D[\alpha]$ be a hypersurface of degree $p$. Then if $g$ is nondegenerate of degree $p$, we have

$$
o\left(T_{f}\left(r, r_{0}\right)\right)+N_{f}\left(r, r_{0}, D\right) \geqslant \int_{\mathbf{C}^{m}\langle r\rangle} \log \left(\eta_{p}(\alpha \circ \varphi) \circ g\right) \sigma .
$$

Proof. Set $\beta=\alpha \circ \varphi$. If $\beta \equiv 0$ then (4.4) is trivially true. Suppose $\beta \neq 0$. We have $u^{p} \alpha \circ \mathfrak{b}=\beta \circ \mathrm{g}$. Set $\tilde{D}=D[\beta]$. Then (2.2) implies

$$
N_{f}\left(r, r_{0}, D\right)+p N_{u}\left(r, r_{0}, 0\right)=N_{g}\left(r, r_{0}, \tilde{D}\right) \text {. }
$$

Therefore

$$
o\left(T_{g}\left(r, r_{0}\right)\right)+N_{f}\left(r, r_{0}, D\right)=N_{g}\left(r, r_{0}, \tilde{D}\right) .
$$

Let $\beta=\Sigma_{J \in K} \beta_{J} \zeta^{J}$ then $\beta \circ g=\Sigma_{J \in K} \beta_{J} g^{J}$ where $g^{J}=\prod_{h=0}^{N} g_{h}^{j_{h}}$. Denote by $D^{J}$ the hypersurface of degree $p$ such that $\operatorname{supp} D^{J}=\mathbf{P}\left(\left(\zeta^{J}\right)^{-1}(0)\right)$. Then by (2.2), we get

$$
\begin{aligned}
N_{g}\left(r, r_{0}, D^{J}\right) & =\sum_{k=0}^{N} j_{k}\left(\int_{\mathbf{C}^{m}\langle r\rangle} \log \left|g_{k}\right| \boldsymbol{\sigma}-\int_{\mathbf{C}^{m}\langle r\rangle} \log \left|g_{k}\right| \boldsymbol{\sigma}\right) \\
& =\sum_{k=0}^{N} j_{k} N_{g}\left(r, r_{0}, H_{k}\right)=o\left(T_{g}\left(r, r_{0}\right)\right)
\end{aligned}
$$

for every $J \in K$. 
If $K(\beta)=\left\{J \in K \mid \beta_{J} \neq 0\right\}$ let $K(\beta)=\left\{J_{0}, \ldots, J_{t}\right\}$ then $\beta \circ \mathrm{g}=\sum_{s=0}^{t} \beta_{J_{s}} g^{J_{s}}$. Set $\tilde{\mathfrak{h}}=\left(g^{J_{0}}, \ldots, g^{J_{t}}\right): \mathbf{C}^{m} \rightarrow \mathbf{C}^{t+1}$ and $h=\mathbf{P}(\tilde{\mathfrak{h}})$. Since $g$ is nondegenerate of degree $p$ then $h$ is nondegenerate. Let $\mathfrak{h}=\left(h_{0}, \ldots, h_{t}\right)$ be a reduced representation of $h$ and $v$ a holomorphic function such that $\tilde{\mathfrak{h}}=v \mathfrak{h}$. We have $g^{J_{s}}=v h_{s}$ for $s=0, \ldots, t$. Let $\tilde{H}_{0}, \ldots, \tilde{H}_{t}$ be the coordinate hyperplanes in $\mathbf{P}_{t}$. Then we have

$$
\begin{aligned}
N_{g}\left(r, r_{0}, D^{J_{s}}\right) & =N_{h}\left(r, r_{0}, \tilde{H}_{s}\right)+N_{v}\left(r, r_{0}, 0\right) \quad \text { for } s=0, \ldots, t, \\
N_{g}\left(r, r_{0}, \tilde{D}\right) & =N_{h}\left(r, r_{0}, \tilde{H}_{t+1}\right)+N_{v}\left(r, r_{0}, 0\right),
\end{aligned}
$$

where $H_{t+1}$ is the hyperplane in $\mathbf{P}_{t}$ given by $\sum_{s=0}^{t} \beta_{J_{s}} z_{s}=0$. If $t=0$ then

$$
\int_{\mathbf{C}^{m}\langle r\rangle} \log \left(\eta_{\rho}(\alpha \circ \varphi) \circ \mathfrak{g}\right) \sigma \leqslant o\left(T_{f}\left(r, r_{0}\right)\right)
$$

and (4.4) is true. Let $t \geqslant 1$. Then proceeding as in the proof of (4.2) of Proposition 4.3 we get

$$
T_{h}\left(r, r_{0}\right) \leqslant \sum_{s=0}^{t+1} N_{h}\left(r, r_{0}, H_{s}\right)+O\left(\log r T_{h}\left(r, r_{0}\right)\right) .
$$

Since $g$ is transcendental and

$$
T_{h}\left(r, r_{0}\right)+N_{v}\left(r, r_{0}, 0\right)=\int_{\mathbf{C}^{m^{\prime}}\langle r\rangle} \log |\tilde{\mathfrak{h}}| \sigma+O(1) \leqslant T_{g}\left(r, r_{0}\right)
$$

we obtain

$$
\begin{aligned}
\int_{\mathbf{C}^{m}\langle r\rangle} \log |\tilde{\mathfrak{h}}| \boldsymbol{\sigma} & \leqslant N_{g}\left(r, r_{0}, \tilde{D}\right)-(t+1) N_{v}\left(r, r_{0}, 0\right)+o\left(T_{g}\left(r, r_{0}\right)\right) \\
& \leqslant N_{g}\left(r, r_{0}, \tilde{D}\right)+o\left(T_{g}\left(r, r_{0}\right)\right) .
\end{aligned}
$$

Finally from Proposition 4.3 and since

$$
|\tilde{\mathfrak{h}}|=\left(\sum_{J \in K(\beta)}\left|\mathrm{g}^{J}\right|^{2}\right)^{1 / 2} \geqslant c \eta_{p}(\beta) \circ \mathrm{g}
$$

for some positive constant $c$, we get (4.4). Q.E.D.

Theorem 4.6 (Second Main Theorem and Defect Relation). Assume that (A1)-(A3) holds, with g nondegenerate of degree $p$ and $\varphi$ surjective. Let $D_{j}=D\left[\alpha_{j}\right]$ be hypersurfaces of degree $p$ for $j=1, \ldots, q, q \geqslant n+1$. Assume $D_{1}, \ldots, D_{q}$ have normal crossings. Then

$$
\begin{gathered}
(q p-n-1) T_{f}\left(r, r_{0}\right) \leqslant \sum_{j=1}^{q} N_{f}\left(r, r_{0}, D_{j}\right)+o\left(T_{f}\left(r, r_{0}\right)\right), \\
\sum_{j=1}^{q} \delta_{f}\left(D_{j}\right) \leqslant(n+1) / p .
\end{gathered}
$$


Proof. Lemma 4.5 and Proposition 3.5 imply

$$
\begin{aligned}
o\left(T_{f}\left(r, r_{0}\right)\right) & +\sum_{j=1}^{q} N_{f}\left(r, r_{0}, D_{j}\right) \geqslant \int_{\mathbf{C}^{m}\langle r\rangle} \log \left(\prod_{j=1}^{q} \eta_{p}\left(\alpha_{j} \circ \varphi\right) \circ \mathfrak{g}\right) \sigma \\
& \geqslant \int_{\mathbf{C}^{m}\langle r\rangle} \log \left(\left|g_{\tau(0)}\right| \cdots\left|g_{\tau(n)}\right||\mathfrak{g}|^{q p-n-1}\right) \boldsymbol{\sigma}+O(1) \\
& =\sum_{j=0}^{n} N_{g}\left(r, r_{0}, H_{\tau(j)}\right)+(q p-n-1) T_{g}\left(r, r_{0}\right)+O(1) .
\end{aligned}
$$

Since $N_{g}\left(r, r_{0}, H_{\tau(j)}\right)=o\left(T_{g}\left(r, r_{0}\right)\right)$ for $j=0, \ldots, n$, then Proposition 4.3 implies (4.5).

From (4.5) we get

$$
\begin{aligned}
\sum_{j=1}^{q} \delta_{f}\left(a_{j}\right) & =\sum_{j=1}^{q} \liminf _{r \rightarrow \infty}\left(1-\frac{N_{f}\left(r, r_{0}, D_{j}\right)}{p T_{f}\left(r, r_{0}\right)}\right) \\
& \leqslant \liminf _{r \rightarrow \infty} \sum_{j=1}^{q}\left(1-\frac{N_{f}\left(r, r_{0}, D_{j}\right)}{p T_{f}\left(r, r_{0}\right)}\right) \\
& \leqslant(n+1) / p . \quad \text { Q.E.D. }
\end{aligned}
$$

REMARK 4.7. In [2] Theorem 4.6 is proved with $g$ and $\varphi$ satisfying weaker conditions, but the proof is much more complicated. So here, also suggested by the referee, is given a weaker version which has a much simpler proof.

5. An example. Let $g: \mathbf{C} \rightarrow \mathbf{P}_{3}$ be the holomorphic map defined by the reduced representation $\mathrm{g}=\left(1, e^{t}, e^{2 t}, e^{3 t}\right): \mathbf{C} \rightarrow \mathbf{C}^{4}$. We have that $g$ is nondegenerate but is degenerate of degree 2 . Let $\varphi: \mathbf{C}^{4} \rightarrow \mathbf{C}^{3}$ be a surjective linear map defined by the matrix

$$
A=\left(\begin{array}{rrrr}
1 & -2 & 0 & 0 \\
0 & 1 & 1 & 0 \\
0 & 0 & -2 & 1
\end{array}\right)
$$

and let $f=\mathbf{P}(\varphi) \circ g$. We have that $f$ is nondegenerate of degree 2 , but an easy computation shows that $f$ is degenerate of degree 3. Consider $p=2$. Let $D_{j}=D\left[\alpha_{j}\right]$ be conics in $\mathbf{P}_{2}$ for $j=1,2,3$ defined by

$$
\begin{aligned}
& \alpha_{1}=9 \zeta_{0}^{2}+36 \zeta_{0} \zeta_{1}+8 \zeta_{0} \zeta_{2}+16 \zeta_{1}^{2}, \\
& \alpha_{2}=8 \zeta_{0} \zeta_{2}+16 \zeta_{1}^{2}+36 \zeta_{1} \zeta_{2}+9 \zeta_{2}^{2}, \\
& \alpha_{3}=\zeta_{0} \zeta_{1}+\zeta_{1} \zeta_{2}+\zeta_{0} \zeta_{2} .
\end{aligned}
$$

We have that $D_{1}, D_{2}, D_{3}$ have normal crossings. By [12, pp. 94-106] we have $T_{f}\left(r, r_{0}\right)=(3 r) / \pi+O(1)$ and since $\alpha_{1} \circ \varphi \circ \mathfrak{g}=9, \quad \alpha_{2} \circ \varphi \circ \mathfrak{g}=9 e^{6 t}$ and $\alpha_{3} \circ \varphi \circ \mathrm{g}=e^{t}\left(1-3 e^{t}+e^{2 t}-3 e^{3 t}+e^{4 t}\right)$ then

$$
N_{f}\left(r, r_{0}, D_{j}\right)=O(1) \text { for } j=1,2
$$

and

$$
N_{f}\left(r, r_{0}, D_{3}\right)=4 r / \pi+O(1)
$$


Hence

$$
\delta_{f}\left(D_{1}\right)=\delta_{f}\left(D_{2}\right)=1 \text { and } \delta_{f}\left(D_{3}\right)=\frac{1}{3}
$$

so

$$
\sum_{j=1}^{3} \delta_{f}\left(D_{j}\right)=\frac{7}{3}>\frac{3}{2} .
$$

Therefore the condition " $g$ nondegenerate of degree $p$ " in Theorem 4.6 cannot be substituted by the weaker condition " $f$ nondegenerate of degree $p$ ".

6. Appendix. Recall the class of meromorphic maps from $\mathbf{C}^{m}$ to $\mathbf{P}_{n}$ defined by $\mathbf{B}$. Shiffman in [10]. Take $\lambda \in \mathbf{N}$. Denote by $\mathcal{E}_{\lambda}\left(\mathbf{C}^{m}\right)$ the ring of holomorphic function $g$ on $\mathbf{C}^{m}$ of the form

$$
g=\sum_{k=1}^{q} \Phi_{k} \exp P_{k},
$$

where $P_{k}$ are polynomials on $\mathbf{C}^{m}$ of degree at most $\lambda$ and $\Phi_{k}$ are meromorphic functions such that $T_{\Phi_{k}}\left(r, r_{0}\right)=o\left(r^{\lambda}\right)$. We shall use the following notation. Let $f$ and $g$ be real valued functions on $\mathbf{R}\left[r_{0}, \infty\right]$. We write $f(r) \sim g(r)$ if $f(r) g(r)^{-1}$ is bounded above and below by positive finite constants for $r$ sufficiently large.

Definition 6.1. A meromorphic map $f: \mathbf{C}^{m} \rightarrow \mathbf{P}_{n}$ is of special exponential type of $\operatorname{order} \lambda$ if $T_{f}\left(r, r_{0}\right) \sim r^{\lambda}$ and it has a reduced representation $\mathfrak{b} \in\left(\mathcal{E}_{\lambda}\left(\mathbf{C}^{m}\right)\right)^{n+1}$.

Denote by $\Xi_{\lambda}$ the class of all meromorphic maps from $\mathbf{C}^{m}$ to $\mathbf{P}_{n}$ of special exponential type of order $\lambda$ and $\subseteq=\bigcup_{\lambda=1}^{\infty} \Im_{\lambda}$.

Proposition 6.2. With the same notation as above we have $\subseteq \subseteq \Re$.

PRoof. Take $f \in \mathbb{S}_{\lambda}$, then there exists a reduced representation $\mathfrak{v} \in\left(\varepsilon_{\lambda}\left(\mathbf{C}^{m}\right)\right)^{n+1}$ of $f$ such that if $\mathfrak{v}=\left(f_{0}, \ldots, f_{n}\right)$ then

$$
f_{j}=\sum_{k=1}^{q_{j}} \Phi_{j k} \exp P_{j k} \quad \text { for } j=0, \ldots, n .
$$

Denote by $\mathfrak{M}$ the complex vector space of all meromorphic functions on $\mathbf{C}^{m}$. Let $\mathfrak{I} \subseteq \mathfrak{M}$ be the subspace spanned by

$$
T=\left\{\Phi_{j k} \exp P_{j k} \mid j=0, \ldots, n \text { and } k=1, \ldots, q_{j}\right\} .
$$

Let $\Upsilon^{\prime} \subseteq \mathfrak{I}$ be the subspace spanned by $f_{0}, \ldots, f_{n}$. Consider $\left\{g_{0}^{\prime}, \ldots, g_{t}^{\prime}\right\} \subseteq T$ a base for $₹$. Let $0 \leqslant j_{0}<\cdots<j_{N} \leqslant t$ be such that $g_{j_{0}}^{\prime}, \ldots, g_{j_{N}}^{\prime}$ is a minimal system of generators for $\mathcal{I}^{\prime}$, i.e. for any $h=\sum_{j=0}^{t} a_{j} g_{j}^{\prime} \in \mathcal{I}^{\prime}$ we have $a_{j}=0$ for $j \in \mathbf{Z}[0, t]-$ $\left\{j_{0}, \ldots, j_{N}\right\}$. Set $\tilde{g}_{k}=g_{j_{k}}^{\prime}, k=0, \ldots, N$.

By definition we have $\tilde{g}_{j}=\Phi_{j} \exp P_{j}$ where $T_{\Phi_{1}}\left(r, r_{0}\right)=o\left(r^{\lambda}\right)$ and $P_{j}$ is a polynomial of degree at most $\lambda$. Since $\Phi_{j}$ is a meromorphic function on $\mathbf{C}^{m}$ there exist $k_{j}$ and $h_{j}$ holomorphic functions on $\mathbf{C}^{m}$ such that $\Phi_{j}=k_{j} / h_{j}$. Let $s_{j}=k_{j} \exp P_{j}$. Then we can choose $k_{j}$ and $h_{j}$ such that $s_{j}$ and $h_{j}$ are coprime. Let $\nu_{j}$ be the 0 -divisor of $h_{j}$. Consider the divisor $\nu$ such that (i) $\operatorname{supp} \nu=\cup_{j=0}^{N} \operatorname{supp} \nu_{j}$; (ii) the multiplicity of every branch $B$ in $\nu$ is equal to the maximum of the multiplicity of $B$ in $\nu_{j}$ for 
$j=0, \ldots, N$. Let $u$ be a holomorphic function such that $u$ has a 0 -divisor $\nu$. Then there exist coprime holomorphic functions $u_{0}, \ldots, u_{N}$ such that $u=u_{j} h_{j}$ for $j=$ $0, \ldots, N$. In addition we have that a holomorphic function $w$ exists such that

$$
h_{0} \cdots h_{N}=w u \text {. }
$$

Then $g_{j}=u \tilde{g}_{j}=u_{j} s_{j}$ is a holomorphic function for $j=0, \ldots, N$. Define $\mathfrak{g}=$ $\left(g_{0}, \ldots, g_{N}\right)$ and $g=\mathbf{P}(g)$. Since $f_{k} \in \mathfrak{I}^{\prime}$ for $k=0, \ldots, n$, we have

$$
u f_{k}=\sum_{j=0}^{N} a_{k}^{j} g_{j}, \quad \text { where } a_{k}^{j} \in \mathbf{C} .
$$

Let $\varphi: \mathbf{C}^{N+1} \rightarrow \mathbf{C}^{n+1}$ be a linear map defined by the matrix $\left(a_{k}^{j}\right)$. Then we have

$$
u \mathfrak{b}=\varphi \circ g \text {. }
$$

In order to prove $f \in \Re$ it remains to check the following.

(6.2) $g$ is nondegenerate.

(6.3) $\mathrm{g}$ is a reduced representation of $g$.

(6.4) $\varphi\left(e_{j}\right) \neq 0$ for $j=0, \ldots, N$.

(6.5) $N_{g}\left(r, r_{0}, H_{j}\right)=o\left(T_{g}\left(r, r_{0}\right)\right)$ for $j=0, \ldots, N$.

(6.6) $N_{u}\left(r, r_{0}, 0\right)=o\left(T_{g}\left(r, r_{0}\right)\right)$.

First we note that $g$ is nondegenerate for the choice of $\tilde{g}_{0}, \ldots, \tilde{g}_{N}$. In order to prove (6.3) we claim that

(6.7) $s_{0}, \ldots, s_{N}$ are coprime.

Suppose $s_{0}, \ldots, s_{N}$ are not coprime. Then there exist holomorphic functions $\mu$, $\tilde{s}_{0}, \ldots, \tilde{s}_{N}$ such that $s_{j}=\mu \tilde{s}_{j}$ for $j=0, \ldots, N$ and $\tilde{s}_{0}, \ldots, \tilde{s}_{N}$ are coprime. Let $B$ be an irreducible branch of the 0 -divisor of $\mu$ and denote by $\mu_{1}$ a holomorphic function which has $B$ as 0 -divisor. Then $\tilde{\mu}=\mu \mu_{1}^{-1}$ is a holomorphic function and

$$
u f_{k}=\sum_{j=0}^{N} a_{k}^{j} u_{j} s_{j}=\mu_{1} \tilde{f}_{k} \text { for } k=0, \ldots, n,
$$

where $\tilde{f}_{k}=\tilde{\mu} \sum_{j=0}^{N} a_{k}^{j} u_{j} \tilde{s}_{j}$. Since $s_{j}$ and $h_{j}$ are coprime, for $j=0, \ldots, N$, then $\mu_{1}$ does not divide $h_{j}$ for $j=0, \ldots, N$ and so $\mu_{1}$ does not divide $u$. Hence $\mu_{1}$ must divide $f_{k}$ for $k=0, \ldots, n$. This implies that $\mathfrak{b}$ is not a reduced representation of $f$. Contradiction. Therefore we have (6.7).

ProOF OF (6.3). Suppose $g_{0}, \ldots, g_{N}$ are not coprime. Then there exist holomorphic functions $\eta, \hat{g}_{0}, \ldots, \hat{g}_{N}$ such that $g_{j}=\eta \hat{g}_{j}$ for $j=0, \ldots, N$ and $\hat{g}_{0}, \ldots, \hat{g}_{N}$ are coprime. Let $B$ be an irreducible branch of the 0-divisor of $\eta$ and denote by $\eta_{1}$ a holomorphic function which has $B$ as 0 -divisor. Then $\tilde{\eta}=\eta \eta_{1}^{-1}$ is a holomorphic function. Since $\eta_{1}$ divides $g_{j}=u_{j} s_{j}$ for $j=0, \ldots, N$, we have that $\eta_{1}$ must divide either $u_{j}$ or $s_{j}$. But $u_{0}, \ldots, u_{N}$ and $s_{0}, \ldots, s_{N}$ are respectively coprime, so there exist $j_{0}, j_{1} \in \mathbf{Z}[0, N]$ such that $\eta_{1}$ does not divide $u_{j_{0}}$ and $s_{j_{1}}$. Hence $\eta_{1}$ must divide $s_{j_{0}}$ and $u_{j_{1}}$. Since $\eta_{1}$ divides $u_{j_{1}}$ but not $u_{j_{0}}$ then $\eta_{1}$ divides $u$ and $h_{j_{0}}$, which implies that $s_{j_{0}}$ and $h_{j_{0}}$ are not coprime. Contradiction. Hence $\mathrm{g}$ is reduced.

Since $\varphi\left(e_{j}\right)=\left(a_{0}^{j}, \ldots, a_{n}^{j}\right)$ then $\varphi\left(e_{j}\right)=0$ implies that $\left\{\tilde{g}_{0}, \ldots,\left(\tilde{g}_{j}\right)^{v}, \ldots, \tilde{g}_{N}\right\}$ is a system of generators for ' $\mathfrak{I}^{\prime}$, which is in contradiction with the minimality of $\left\{\tilde{g}_{0}, \ldots, \tilde{g}_{N}\right\}$. Therefore we have (6.4). 
Since $T_{g}\left(r, r_{0}\right) \sim r^{\lambda}$ and $T_{\Phi_{j}}\left(r, r_{0}\right)=o\left(r^{\lambda}\right)$, then (2.1) and (6.1) imply

$$
N_{u}\left(r, r_{0}, 0\right)=o\left(r^{\lambda}\right)=o\left(T_{g}\left(r, r_{0}\right)\right)
$$

and

$$
\begin{aligned}
N_{g_{j}}\left(r, r_{0}, 0\right) & =N_{u_{j}}\left(r, r_{0}, 0\right)+N_{k_{j}}\left(r, r_{0}, 0\right) \\
& \leqslant N_{u}\left(r, r_{0}, 0\right)+T_{\Phi_{j}}\left(r, r_{0}\right)=o\left(T_{g}\left(r, r_{0}\right)\right)
\end{aligned}
$$

for $j=0, \ldots, N$. Hence (6.5) and (6.6) are proved. Q.E.D.

\section{REFERENCES}

1. L. V. Ahlfors, The theory of meromorphic curves, Acta Soc. Sci. Fenn. (Nova Ser.) 3 (1941), 1-31.

2. A. Biancofiore, A hypersurface defect relation for a class of meromorphic maps, Thesis, Univ. of Notre Dame, 1981.

3. J. Carlson and P. A. Griffiths, $A$ defect relation for equidimensional, holomorphic mappings between algebraic varieties, Ann. of Math. 95 (1972), 557-584.

4. H. Cartan, Sur les zéros des combinaisons linéaires de p fonctions holomorphes données, Mathematica (Cluj) 7 (1933), 80-103.

5. P. A. Griffiths, Holomorphic mappings: Survey of some results and discussion of open problems, Bull. Amer. Math. Soc. 78 (1972), 374-382.

6. P. A. Griffiths and J. King, Nevanlinna theory and holomorphic mappings hetween algebraic varieties, Acta Math. 130 (1973), 145-220.

7. S. Mori, On the deficiencies of meromorphic mappings of $\mathbf{C}^{n}$ into $\mathbf{P}^{N} \mathbf{C}$, Nagoya Math. J. 67 (1977), $165-176$.

8. R. Nevanlinna, Le théorème de Picard-Borel et la théorie des fonctions meromorphes, Gauthier-Villars, Paris, 1929.

9. B. Shiffman, Holomorphic curves in algebraic manifolds, Bull. Amer. Math. Soc. 83 (1977), 553-568.

10. . On holomorphic curves and meromorphic maps in projective space, Indiana Univ. Math. J. 28 (1979), 627-641.

11. W. Stoll, Die beiden Hauptsätze der Wertverteilungstheorie bei Funktionen mehrer komplexen Veründerlichen (I), (II), Acta Math. 90 (1953), 1-115; 92 (1954), 55-169.

12. A. Vitter, The lemma of the logarithmic derivative in several complex variables, Duke Math. J. 44 (1977), 89-104.

13. H. Weyl and F. J. Weyl, Meromorphic functions and analytic curves, Princeton Univ. Press, Princeton, N.J., 1943.

Department of Mathematics, University of Notre Dame, Notre Dame, Indiana 46556 